\title{
Three-dimensional core-shell ferromagnetic nanowires grown by focused electron beam induced deposition
}

\author{
Javier Pablo-Navarro ${ }^{1,2}$, César Magén ${ }^{1,3,4^{*}}$ and José María de Teresa ${ }^{1,2,3}$
}

\author{
${ }^{1}$ Laboratorio de Microscopías Avanzadas (LMA) - Instituto de Nanociencia de Aragón (INA), \\ Universidad de Zaragoza, 50018 Zaragoza, Spain. \\ ${ }^{2}$ Instituto de Ciencia de Materiales de Aragón (ICMA), Universidad de Zaragoza- CSIC, 50009 \\ Zaragoza, Spain. \\ ${ }^{3}$ Departamento de Física de la Materia Condensada, Universidad de Zaragoza, 50009 Zaragoza, \\ Spain. \\ ${ }^{4}$ Fundación ARAID, 50018 Zaragoza, Spain.
}

\begin{abstract}
Functional nanostructured materials often rely on the combination of more than one material to confer the desired functionality or an enhanced performance of the device. Here we report the procedure to create nanoscale heterostructured materials in the form of core-shell nanowires by focused electron beam induced deposition (FEBID) technologies. In our case, three-dimensional nanowires $(<100 \mathrm{~nm}$ in diameter) with metallic ferromagnetic cores of Co- and Fe-FEBID have been grown and coated with a protective Pt-FEBID shell (ranging 10-20 nm in thickness) aimed to minimize the degradation of magnetic properties caused by the surface oxidation of the core to a non-ferromagnetic material. The structure, chemistry and magnetism of nanowire cores of Co and Fe have been characterized in Pt-coated and uncoated nanostructures to demonstrate that the morphology of the shell is conserved during Pt coating, the surface oxidation is suppressed or confined to the Pt layer, and the average ferromagnetism of the core is strengthened up to
\end{abstract}


$30 \%$. The proposed approach paves the way to the fabrication of three-dimensional FEBID nanostructures based on the smart alternate deposition of two or more materials combining different physical properties or added functionalities.

Keywords: magnetic nanowires, core-shell structures, focused electron beam induced deposition, cobalt, iron, transmission electron microscopy, electron holography

*Author to whom correspondence should be addressed:

César Magén (cmagend@unizar.es)

Laboratorio de Microscopías Avanzadas (LMA)

Instituto de Nanociencia de Aragón (INA)

Universidad de Zaragoza

C/ Mariano Esquillor, Edificio I+D

50018 Zaragoza - SPAIN - EU

Tel: +34 876555369

Fax: +34 976762776 


\section{INTRODUCTION}

Focused electron beam induced deposition (FEBID) has demonstrated to be a successful nanofabrication technology with outstanding present and future application [1-7]. This singlestep nanolithography method enables the fabrication of two-dimensional (2D) and threedimensional (3D) nano-objects from a growing catalogue of chemical elements and compounds, comprising a great variety of physical properties such as metallicity and magnetoresistance,[8] insulating behavior [9], superconductivity [10], ferromagnetism and superparamagnetism [11,12], plasmonic behavior [13], etc. FEBID consists in the injection of a suitable precursor gas, usually an organometallic compound, on the deposition chamber with a gas injector system (GIS), and the local decomposition of these gas molecules using a scanning electron beam focused on the surface. FEBID has been remarkably successful in growing nanometer-scale objects and devices with complex 2D or 3D geometries [14-23], even on unusual substrates such as scanning probe tips $[11,24,25]$. However, as this technique is based on the local decomposition of gas molecules containing the targeted element, the presence of impurities derived from precursor residues has been a central issue in FEBID. As a consequence, significant efforts have been devoted to deposit purification [26-30]. For magnetism-based applications, the use of Co-based and Fe-based carbonyl precursors gives rise to FEBID structures with metal purities $>80 \%$ for Co [18,31,32] and Fe [33-36]. This has enabled the fabrication of numerous functional devices based on FEBID magnetic elements [11,18-20,37] and, only in the case of magnetic deposits with initial low magnetic content $(<80 \%)$, it makes sense to perform purification processes [38].

One of the future challenges for FEBID technology is the fabrication of heterogeneous structures combining the properties of more than one material. Limited attempts have been reported in this direction, such as the use of double-precursor deposition producing phase segregation [39], the growth of 2D multilayers with alternate deposition [40], the coverage of an array of nanopillars with a 2D layer [41] and the creation of magnetic-superconducting nanocontacts [42]. Functional nanoscale devices are most often heterostructures composed of layers of different materials with the aim of adding multiple or new functionalities, improving 
the performance, and/or protecting from oxidation or corrosion. On the one hand, heterostructured materials combining different properties (ferromagnetic/non-magnetic, metallic/insulator, ferromagnetic/superconducting, ferromagnetic-plasmonic, etc.) may present either multi-functionality or additional functional interface properties. As an example of the latter, we can cite the emergence of a 2D high-mobility electron gas forming at the interface of two insulating films [43] or the emergence of ferromagnetism or superconductivity at the interface of two materials which do not exhibit such properties [44]. On the other hand, surface exposure to air can degrade the properties of objects with a high surface-to-volume ratio, such as nanoparticles or nanowires (NWs), where the surface exposed to ambient atmosphere may represent a significant volume fraction of the whole object, and show different structural, magnetic and transport properties with respect to the inner volume. This is particularly true for ferromagnetic nanostructures such as cobalt and iron, which present natural surface oxidation layers of a few nanometers degrading or completely modifying their magnetic behavior $[36,45]$. Therefore, the fabrication of heterogeneous materials shows great potential across many research fields, remaining nearly unexplored in the case of FEBID method. In this work we report the sequential deposition of two different materials to produce three-dimensional coreshell NWs. Core-shell NWs are functional nanostructures with applications in several domains such as semiconductor-based elements for electronic devices [46] and photovoltaic applications [47], constituents of long-life lithium battery electrodes [48] and supercapacitors [49], efficient light absorbers in solar cells [50], etc. Very few examples of successful growth of magnetic core-shell NWs by electrodeposition technique can be found in literature [51,52]. Such magnetic core-shell NWs are expected to find applications in magnetic memories, sensors, multiferroic devices, magnetoplasmonics, magnetic hyperthermia, etc.

To demonstrate the feasibility and versatility of FEBID to grow core-shell NWs, we have fabricated core-shell ferromagnetic NWs made of Co and Fe core, and coated them with Ptbased layers aiming to suppress surface oxidation and maximize the magnetic response of the magnetic core. The effectiveness of the Pt shell is demonstrated by transmission electron microscopy (TEM) and electron holography characterization, which reveals the confinement of 
the oxidation in the Pt layer, protecting completely the magnetic core, and the subsequent increase of the average magnetization of the Co and Fe cores. The successful application of FEBID to grow three-dimensional core-shell magnetic NWs opens a new path to engineer the physical properties of magnetic FEBID deposits. Indeed, the approach used is general and can be applied to any pair of materials that can be grown by the FEBID technique, enlarging its interest.

\section{METHODS}

\section{a) Growth of Co, Fe and Pt nanostructures by FEBID.}

The specimens were fabricated in the commercial Helios Nanolab 650 Dual Beam and Helios Nanolab 600 Dual Beam systems equipped with a Schottky field emission gun (S-FEG) electron column, a $\mathrm{Ga}^{+}$ion column and GIS for the deposition of $\mathrm{Co}, \mathrm{Fe}$ and Pt. The substrates were $\mathrm{Cu}$ TEM grids to facilitate the analysis. The gas precursor gases used were $\mathrm{Co}_{2}(\mathrm{CO})_{8}, \mathrm{Fe}_{2}(\mathrm{CO})_{9}$, and $\mathrm{CH}_{3} \mathrm{CpPt}\left(\mathrm{CH}_{3}\right)_{3}$. The main deposition parameters (beam current and working pressure) were optimized both in Co and Fe cases. FEBID-Co deposits were grown with electron beam currents ranging from $50 \mathrm{pA}$ to $800 \mathrm{pA}$ at a base pressure of $1 \times 10^{-6} \mathrm{mbar}$, and working gas pressures ranging from $6 \times 10^{-6}$ to $1 \times 10^{-5}$ mbar at $100 \mathrm{pA}$. As illustrated in Figure S1(a) of the Supplementary Data, for the maximum working pressure available $\left(1 \times 10^{-5} \mathrm{mbar}\right)$, the Co content increases remarkably with the beam current, from approximately $60 \%$ at. for $25 \mathrm{pA}$ to $85 \%$ at. for $800 \mathrm{pA}$. It is also observed that for a fixed beam current of $100 \mathrm{pA}$, the cobalt content decreases with the increasing working pressure, see Figure S1(b). Figure S1(c) and (d) illustrate the Fe-FEBID case, where Fe content at constant working pressure of $1 \times 10^{-5}$ mbar remains nearly constant around $75 \%$ at. within the experimental error as a function of the beam current.

\section{b) (Scanning) Transmission Electron Microscopy characterization.}

High resolution transmission electron microscopy imaging of the NWs was carried out in an image-corrected FEI Titan Cube 60-300 operated at $300 \mathrm{kV}$ and equipped with a S-FEG and a CETCOR corrector for the objective lens from CEOS, providing a point resolution in TEM 
mode below $1 \AA$, and a bottom-mounted $2 \mathrm{~K}$ x 2K Ultrascan CCD camera from Gatan. Scanning transmission electron microscopy (STEM) imaging and electron energy loss spectroscopy (EELS) were carried out in a probe-corrected Titan Low Base 60-300 operated at $300 \mathrm{kV}$ and equipped with a high brightness Schottky field emission gun, a CETCOR corrector for the condenser system to provide sub-angstrom probe size, and a Gatan Image Filter (GIF) Tridiem 866 ERS for EELS analysis. Both imaging and spectroscopic experiments were carried out with a convergence semi-angle of $25 \mathrm{mrad}$. STEM-EELS spectrum imaging experiments were carried out with an energy dispersion of $0.8 \mathrm{eV}$, the energy resolution of the EELS experiments was approximately $1.5 \mathrm{eV}$, the pixel time was $35 \mathrm{~ms}$ and the beam current $\sim 250 \mathrm{pA}$.

\section{c) Electron holography experiments.}

The magnetization of the core-shell NWs synthesized was analyzed by off-axis electron holography carried out in the Titan Cube described above at $300 \mathrm{kV}$. For this purpose, this instrument is equipped with a motorized electrostatic biprism and a Lorentz lens. The experiments were performed in Lorentz mode (with the objective lens switched off, and the Lorentz lens operating as the image-forming lens). In the holographic experiments, the excitation of the biprism was varied between 180 and $220 \mathrm{~V}$, depending on the actual diameter of the NWs, to produce holograms with a fringe contrast ranging $20-25 \%$. The acquisition time of the holograms was $5 \mathrm{~s}$. The method to extract the remanent magnetic state of the NWs is described in detail in the Supplementary Data.

\section{RESULTS AND DISCUSSION}

The starting point for the synthesis of the nanostructures is the growth of Co and Fe NWs by FEBID following the procedures described in previous works [21,53]. The optimal growth parameters in order to obtain a moderate diameter $(<100 \mathrm{~nm})$ and the highest possible metallic content for the core material have to be determined. Among the numerous parameters affecting the physical properties of the nanodeposits, we have explored those considered most important: the flux of the gas precursor injected and the beam current, which affect both the diameter of the deposit and the metallic content. 
We have observed that thinner NWs are favored by high fluxes and low beam currents. Small diameters are linked to a low temperature of the nanostructures during the growth and a small beam diameter. The cooling is achieved both using large contact heat dissipation, which is produced using a large number of gas precursor molecules hitting the NW, and low currents. Besides, the reduction of the beam diameter, which is proportional to the square root of the current, is accomplished by a beam current decrease [18]. High metallic content is associated with moderate fluxes and high beam currents [31]. Firstly, the metallic content increases with the decreasing gas pressure because in high gas fluxes the precursor is refreshed too quickly to allow proper decomposition and non-metallic residues are added to the deposit. As the flux is reduced the metal deposition purity is improved [54]. However, the pressure decrease comes to a point where mostly carbon and oxygen are deposited and purity diminishes. Secondly, high beam currents raise the temperature contributing to the thermal dissociation of the precursor gas [55], and at the same time, desorption of volatile molecules is favored [56]. In addition, when the temperature gets higher, new crystallization processes take place and different structural shapes appear. The Co NWs exhibit a smooth surface when they have low metallic purity (approximately $<70 \%$ ), but small roughness can be noticed (with diameter variations in the range of 5 nanometers) for higher metallic contents. Fe NWs present a similar behavior at the highest metallic contents. However, roughness here is clearly caused by the preferential growth of grains at the surface of the NW. This effect is especially remarkable in the thinnest Fe NWs, where the diameter variation can occasionally reach $30 \%$ of the average diameter. Thus, roughness can be used as an indirect qualitative indication of the metallic purity.

Considering the increasing dependence of the NW diameter with the current and its reduction upon increasing working pressure, compromise values of $100 \mathrm{pA}$ and $\sim 6 \times 10^{-5}$ mbar have been chosen to obtain NWs with $>85 \%$ Co content and $70 \mathrm{~nm}$ of diameter. The metallic content of Fe-FEBID deposits is less dependent on the beam current and gas pressure. In particular, Fe content at the maximum working pressure remains nearly constant (around $75 \%$ at.) while it slowly decreases upon increasing working pressure for a fixed current. In this case, a current of $86 \mathrm{pA}$ and a working pressure of $\sim 3.3 \times 10^{-6}$ mbar were selected to the core-shell structures with 
$80 \% \mathrm{Fe}$ content and $60 \mathrm{~nm}$ of diameter. Further details about the experimental parameters were provided in the Methods Section.

In order to fabricate 3D core-shell NWs, the procedure described in Figure 1 illustrates the method employed. The cobalt or iron core of the nanostructures was grown in TEM copper grids, where the beam impacts on the substrate mounted on a stage $0^{\circ}$ tilted. Once the magnetic core is grown, the Pt coating was carried out. Firstly, the stage is tilted $52^{\circ}$, so the nanostructures are seen using the electron beam with the same perspective as that in Figure 2. Then a rectangular pattern of Pt deposition is set with the same height and width as the cobalt deposit and a depth of $\sim 10 \mathrm{~nm}$. Finally, the stage is rotated $180^{\circ}$ and a new deposit is performed in the same way, completing the whole coating. Even though only two opposite sides of the NW are patterned with Pt deposition, the secondary electrons produced induce a complete coverage of the magnetic cores. This leads to a $\sim 20 \mathrm{~nm}$ increase of the diameter as can be certified in the cross sectional image of one of the core-shell NWs, shown in Figure 2. For experimental comparison, in all cases two identical cores (grown with the same experimental conditions) were grown one immediately after the other. Then, one of them was coated with Pt-FEBID, while the other was kept as a reference.

Figure 2 shows SEM images of Co and Fe NWs before coating (a, c) and after Pt coating (b, d). The Co and Fe NWs grow straight with uniform diameter. The Pt coating enlarges the diameter of the NWs and smooths out the initial roughness of the Co and Fe NWs as noticed after careful inspection of the images in Figure 2. In order to investigate the effectiveness and homogeneity of the Pt coverage, cross sections perpendicular to the NW axis were prepared using focused ion beam methods for TEM lamella preparation (see Section 2 of the Supplementary Data). Such cross sections were subsequently investigated by means of STEM-EELS chemical maps. The results obtained for the Pt-coated Co-FEBID nanowire shown in Figure 2(b) are illustrated in Figure 3. We can observe that the Co core section is approximately circular, while the Pt completely wraps the Co core with a $10 \mathrm{~nm}$-thick shell, forming a protective layer. The Pt shell is subsequently covered by a $\mathrm{W}$ layer and an additional Pt layer deposited to protect the nanowires during lamella preparation. To our understanding, this is the first growth of a 
cylindrical core-shell NW by FEBID via sequential deposition of the core and the shell targeted materials.

High-resolution transmission electron microscopy (HRTEM) images shown in Figure 4 describe with more detail the microstructure of these structures and confirm the core-shell nature of the coated ones. The uncoated nanostructures present the expected nanocrystalline microstructure already reported for these materials when they present high metallic content [57], see Supplementary Data. In 2D objects nanocrystals of 2-3 nm were reported, which in this case are harder to observe doe to the large diameter of the NW in comparison with standard cross sectional TEM specimens. No texture is observed all along the nanowire. Interestingly, both Co and $\mathrm{Fe}$ uncoated specimens present a surface layer of $\sim 5 \mathrm{~nm}$ with brighter contrast, layer whose nanocrystalline nature is more easily observable with respect to the inner NW, which represents the natural oxidation layer. This is confirmed by the compositional analysis shown afterwards. In the Pt-coated NWs, the core contrast is a bit masked by the intense contribution from the distribution of Pt particles (less than $5 \mathrm{~nm}$ of diameter) contained in the amorphous matrix of PtFEBID and covering the core NWs in all directions.

Chemical mapping provides further insight about the new core-shell nanostructures. Figure 4 also shows, accompanying their correspondent HRTEM image, a comparative of chemical mapping experiments carried out in NWs with the same core size for both Co and Fe. Such quantitative compositional mapping has been carried out by means of Electron Energy Loss Spectroscopy (EELS) in Scanning TEM (STEM) mode. Firstly, these maps confirm that the surface layer observed in the naked NWs indeed correspond to an oxidation of the metallic Co or $\mathrm{Fe}$, represented by orange-colored regions in the maps. It extends to approximately $5 \mathrm{~nm}$, which corresponds nicely with the lighter-contrast surface observed in HRTEM. Further proof that the high oxygen content is related to natural oxidation upon exposure to ambient air, is the fact that in the Fe case, with a significantly larger roughness, the oxide layer contours perfectly the irregularities of the surface, penetrating regularly about $4 \mathrm{~nm}$ into the NW. To give a better account on the location of oxygen in these nanostructures, Figure 5 plots STEM-EELS line profiles of integrated intensities of the edges of the different elements, to show how the oxygen 
signal does not coincide anymore with the $\mathrm{Co}(\mathrm{Fe})$ signal once the NWs are coated, while there is a clear coincidence between the surface decaying metal signal and the oxygen when they are directly exposed to air. Thus, the growth of a thin FEBID-Pt layer fully protects the magnetic core, avoiding its oxidation.

This has a significant impact on the magnetic properties of the whole NW structure as a whole. Off-axis electron holography (EH) has been applied to explore the local magnetic properties of the NWs and to analyze the influence of Pt-coating on the magnetism of the Co and Fe NWs $[18,53]$. EH enables to extract the magnetic phase shift induced by the local electromagnetic fields created by a material at the nanoscale [58]. Following the procedure described in the Methods section, magnetic induction maps and flux line distributions can be quantitatively extracted in the remanent state of the NWs. As a result, Figure 6(a-d) plot the magnetic flux distribution obtained in both types of core-shell NWs, compared with the uncoated ones, showing the expected monodomain-type magnetization distribution pointing along the NWs axis due to the high shape anisotropy of the nanostructure. These maps have been extracted from central regions of the NWs, far from the tip or any other major defect to help quantifying the net magnetic induction produced by the core of the NWs in the coated and uncoated cases. To do this, a cylindrical shape with uniform magnetization along the nanowire longitudinal axis has been assumed for the NWs in all cases, in order to probe the influence of the oxidized shell in the total net magnetic induction of the structure. Thus, if $z$ is the optical axis (the direction of the electron beam), $x$ is the nanowire axis (horizontal) and $y$ is perpendicular to $x$ and $z$, the average magnetic induction is $B_{x}$. Following the electron holography method described in the Supplementary Data, $B_{x}$ can be calculated as:

$$
\left|B_{x}(x, y)\right|=\frac{\hbar}{e \cdot t} \frac{\partial \varphi_{M A G}(x, y)}{\partial y}
$$

where $\hbar$ is the reduced Planck constant, $\varphi_{M A G}$ the magnetic component which contribute to the electron phase shift $\varphi(\vec{r}), e$ the electron charge and $t$ the magnetic thickness of the specimen. The magnetic induction obtained is the average total value of the core NW. This implies that in 
the case of the uncoated NWs, the oxide layer contributes to reduce this magnetic induction value.

The comparison of this magnitude for both coated and uncoated NWs in shown in Figure 6(e-f), and gives rise to a systematic increase of the magnetic induction in the case of the core shells with respect to the naked cores, confirming that the oxidized shell is not or weakly ferromagnetic. The enhancement of the magnetic induction is moderate in Co, about $17 \%$ in the NW with a 70-nm-wide Co core, and increases up to $35 \%$ in the case of Fe NWs. The latter are much thinner (Fe core is about $35 \mathrm{~nm}$ of diameter) and thus their overall magnetic properties are much more affected by surface oxidation than thicker Co NWs. Both are still below the bulk values due to the residues present in FEBID deposits, which in this case reduces the Co and Fe content down to $85 \%$ and $75 \%$, respectively.

\section{DISCUSSION}

This new synthetic method of core-shell NWs by FEBID technologies opens a new path for the fabrication of (multi)functional nanostructures through the engineering of physical properties by tailored designed architectures based on a smart combination of FEBID materials, thicknesses and geometries [13-22]. In the case of magnetic materials explored here as proof-of-concept, the magnetization enhancement could have a significant impact in magnetic devices grown by FEBID and nanostructures at such small scale, such as nanosensors, high-density magnetic memories or functionalized MFM tips, whose magnetic or electric response can be much affected by the degradation of the magnetic properties of the outer surface when it becomes a significant fraction of their volume. These application would require additional characterization, such as the measurement of the magnetotransport experiments of individual NWs, which would provide key information on the magnetic behavior of these systems $[59,60]$. Furthermore, coreshell bimagnetic nanostructures could be grown in which basic magnetic properties such as coercivity or remanence can be fine-tuned by alternating two different magnetic materials or inserting non-magnetic spacing layers. Furthermore, materials with different (even excluding) properties could be combined to explore novel phenomena or proximity effects at the nanoscale. 
For instance, exotic high-velocity magnetic states could set in cylindrical ferromagnetic nanowires (nanotubes) grown around non-magnetic cores [61]. Also superconducting W-based FEBID nanowires [10] and ferromagnetic FEBID-Co(Fe) ones could be integrated to produce superconducting-ferromagnetic core-shell nanostructures as playground for the investigation of the interplay between ferromagnetism and superconductivity in three dimensional confined nanostructures. In addition, the combination of noble metals (i.e. FEBID-Au) $[12,13]$ and ferromagnetic FEBID materials to investigate magnetoplasmonic responses in nanoscaled coreshell devices could be challenged. An almost infinite combinations of materials and thicknesses could be explored, opening new prospects in the applicability of FEBID technologies in the fabrication of functional nanodevices. Further opportunities could arise from the optimization of the method presented in this work, such as the application of a continuous rotation of the core during shell deposition. This may also include the combination of FEBID technologies with other synthesis or nanolithography techniques, for instance the atomic layer deposition of a wide variety of materials on FEBID-grown seeds, which could produce new (multifunctional) nanostructures enjoying the remarkable advantages of this deposition technique.

\section{CONCLUSION}

In summary, we have demonstrated that FEBID technology can be applied to fabricate threedimensional nanowires where ultrathin ferromagnetic cores of Co- and Fe-FEBID, down to 35 $\mathrm{nm}$ in diameter, are successfully protected from oxidation by $10-20 \mathrm{~nm}$ thick Pt-FEBID shells. As a result, the average magnetization of the NWs has been improved up to $35 \%$ in the thinnest nanowires, 35-nm-thick Fe-FEBID cores, which could have a strong impact in the functional properties of these magnetic nanostructures. The nanofabrication procedures described represents a novel approach to produce a new generation of custom-designed heterostructured functional materials fabricated solely by FEBID or its combination with other synthetic methods.

\section{Acknowledgements}


Financial support from Spanish Ministerio de Economía y Competitividad through the project MAT2014-51982-C2 and from regional Gobierno de Aragón through project E26 with European Social Fund funding is acknowledged. J. P.-N. grant is funded by the Ayuda para Contratos Predoctorales para la Formación de Doctores, Convocatoria Res. 05/06/15 (BOE 12/06/15) of the Secretaría de Estado de Investigación, Desarrollo e Innovación in the Subprograma Estatal de Formación of the Spanish Ministry of Economy and Competitiveness (MINECO) with the participation of the European Social Fund. The authors acknowledge L. Casado and I. Rivas for their invaluable support in the development of the FEBID methods and lamella preparation.

\section{REFERENCES}

[1] van Dorp W F and Hagen C W 2008 A critical literature review of focused electron beam induced deposition J. Appl. Phys. 104081301

[2] Utke I, Hoffmann P and Melngailis J 2008 Gas-assisted focused electron beam and ion beam processing and fabrication J. Vac. Sci. Technol. B Microelectron. Nanom. Struct. 261197

[3] De Teresa J M and Fernández-Pacheco A 2014 Present and future applications of magnetic nanostructures grown by FEBID Appl. Phys. A 117 1645-58

[4] De Teresa J M, Fernández-Pacheco A, Córdoba R, Serrano-Ramón L, Sangiao S and Ibarra M R 2016 Review of magnetic nanostructures grown by focused electron beam induced deposition ( FEBID ) J. Phys. D Appl. Phys. Phys. X X

[5] Randolph S J, Fowlkes J D and Rack P D 2006 Focused, Nanoscale Electron-BeamInduced Deposition and Etching Crit. Rev. Solid State Mater. Sci. 31 55-89

[6] Hagen C W 2014 The future of focused electron beam-induced processing Appl. Phys. A 
117 1599-605

[7] Bret T, Hofmann T and Edinger K 2014 Industrial perspective on focused electron beam-induced processes Appl. Phys. A 117 1607-14

[8] Huth M, Porrati F, Schwalb C, Winhold M, Sachser R, Dukic M, Adams J and Fantner G 2012 Focused electron beam induced deposition: A perspective Beilstein J. Nanotechnol. 3 597-619

[9] Riazanova A V, Costanzi B N, Aristov A, Rikers Y G M, Ström V, Mulders J J L, Kabashin A V, Dahlberg E D and Belova L M 2014 Gas-assisted electron-beam-induced nanopatterning of high-quality Si-based insulator Nanotechnology 25155301

[10] Sengupta S, Li C, Baumier C, Kasumov A, Guéron S, Bouchiat H and Fortuna F 2015 Superconducting nanowires by electron-beam-induced deposition Appl. Phys. Lett. 106 042601

[11] Utke I, Hoffmann P, Berger R and Scandella L 2002 High-resolution magnetic Co supertips grown by a focused electron beam Appl. Phys. Lett. 804792

[12] Gabureac M, Bernau L, Utke I and Boero G 2010 Granular Co-C nano-Hall sensors by focused-beam-induced deposition Nanotechnology 21115503

[13] Graells S, Aćimović S, Volpe G and Quidant R 2010 Direct Growth of Optical Antennas Using E-Beam-Induced Gold Deposition Plasmonics 5 135-9

[14] Höflich K, Yang R Bin, Berger A, Leuchs G and Christiansen S 2011 The Direct Writing of Plasmonic Gold Nanostructures by Electron-Beam-Induced Deposition $A d v$. Mater. 23 2657-61

[15] Winkler R, Szkudlarek A, Fowlkes J D, Rack P D, Utke I and Plank H 2015 Toward Ultraflat Surface Morphologies During Focused Electron Beam Induced Nanosynthesis: Disruption Origins and Compensation ACS Appl. Mater. Interfaces 7 3289-97

[16] Mitsuishi K, Shimojo M, Tanaka M, Takeguchi M and Furuya K 2005 Resolution in 
New Nanofabrication Technique Combining Electron-Beam-Induced Deposition and Low-Energy Ion Milling Jpn. J. Appl. Phys. 44 5627-30

[17] Fedorov A G, Kim S, Henry M, Kulkarni D and Tsukruk V V. 2014 Focused-electronbeam-induced processing (FEBIP) for emerging applications in carbon nanoelectronics Appl. Phys. A 117 1659-74

[18] Serrano-Ramón L, Córdoba R, Rodríguez L A, Magén C, Snoeck E, Gatel C, Serrano I, Ibarra M R and De Teresa J M 2011 Ultrasmall functional ferromagnetic nanostructures grown by focused electron-beam-induced deposition ACS Nano 5 7781-7

[19] De Teresa J M and Córdoba R 2014 Arrays of densely packed isolated nanowires by focused beam induced deposition plus ar(+) milling. ACS Nano 8 3788-95

[20] Boero G, Utke I, Bret T, Quack N, Todorova M, Mouaziz S, Kejik P, Brugger J, Popovic R S and Hoffmann P 2005 Submicrometer Hall devices fabricated by focused electron-beam-induced deposition Appl. Phys. Lett. 86042503

[21] Fernández-Pacheco A, Serrano-Ramón L, Michalik J M, Ibarra M R, De Teresa J M, O’Brien L, Petit D, Lee J and Cowburn R P 2013 Three dimensional magnetic nanowires grown by focused electron-beam induced deposition Sci. Rep. 31492

[22] van Kouwen L, Botman A and Hagen C W 2009 Focused electron-beam-induced deposition of $3 \mathrm{~nm}$ dots in a scanning electron microscope. Nanoletters 9 2149-52

[23] Gavagnin M, Wanzenboeck H D, Belić D and Bertagnolli E 2013 Synthesis of Individually Tuned Nanomagnets for Nanomagnet Logic by Direct Write Focused Electron Beam Induced Deposition ACS Nano 7 777-84

[24] Belova L M, Hellwig O, Dobisz E and Dan Dahlberg E 2012 Rapid preparation of electron beam induced deposition Co magnetic force microscopy tips with $10 \mathrm{~nm}$ spatial resolution. Rev. Sci. Instrum. $\mathbf{8 3} 093711$

[25] Lavenant H 2014 Mechanical magnetometry of Cobalt nanospheres deposited by 
focused electron beam at the tip of ultra-soft cantilevers Nanofabrication 1 65-73

[26] Botman A, Mulders J J L and Hagen C W 2009 Creating pure nanostructures from electron-beam-induced deposition using purification techniques: a technology perspective. Nanotechnology 20372001

[27] Mehendale S, Mulders J J L and Trompenaars P H F 2015 Purification of Au EBID structures by electron beam post-irradiation under oxygen flux at room temperature Microelectron. Eng. 141 207-10

[28] Belić D, Shawrav M M, Gavagnin M, Stöger-Pollach M, Wanzenboeck H D and Bertagnolli E 2015 Direct-write deposition and focused-electron-beam-induced purification of gold nanostructures ACS Appl. Mater. Interfaces 7 2467-79

[29] Plank H, Noh J H, Fowlkes J D, Lester K, Lewis B B and Rack P D 2014 Electronbeam-assisted oxygen purification at low temperatures for electron-beam-induced $\mathrm{Pt}$ deposits: Towards pure and high-fidelity nanostructures ACS Appl. Mater. Interfaces 6 $1018-24$

[30] Stanford M G, Lewis B B, Noh J H, Fowlkes J D, Roberts N A, Plank H and Rack P D 2014 Purification of Nanoscale Electron-Beam-Induced Platinum Deposits via a Pulsed Laser-Induced Oxidation Reaction ACS Appl. Mater. Interfaces 6 21256-63

[31] Fernández-Pacheco A, De Teresa J M, Córdoba R and Ibarra M R 2009 Magnetotransport properties of high-quality cobalt nanowires grown by focusedelectron-beam-induced deposition J. Phys. D. Appl. Phys. 42055005

[32] Belova L M, Dahlberg E D, Riazanova A, Mulders J J L, Christophersen C and Eckert J 2011 Rapid electron beam assisted patterning of pure cobalt at elevated temperatures via seeded growth Nanotechnology 22145305

[33] Takeguchi M, Shimojo M and Furuya K 2005 Fabrication of magnetic nanostructures using electron beam induced chemical vapour deposition Nanotechnology 16 1321-5 
[34] Lukasczyk T, Schirmer M, Steinrück H-P and Marbach H 2009 Generation of Clean Iron Structures by Electron-Beam-Induced Deposition and Selective Catalytic Decomposition of Iron Pentacarbonyl on Rh(110) Langmuir 25 11930-9

[35] Lavrijsen R, Córdoba R, Schoenaker F J, Ellis T H, Barcones B, Kohlhepp J T, Swagten H J M, Koopmans B, De Teresa J M, Magén C, Ibarra M R, Trompenaars P and Mulders J J L 2011 Fe:O:C grown by focused-electron-beam-induced deposition: magnetic and electric properties Nanotechnology 22025302

[36] Rodríguez L A, Deen L, Córdoba R, Magén C, Snoeck E, Koopmans B and De Teresa J M 2015 Influence of the shape and surface oxidation in the magnetization reversal of thin iron nanowires grown by focused electron beam induced deposition Beilstein J. Nanotechnol. 6 1319-31

[37] Vavassori P, Pancaldi M, Perez-Roldan M J, Chuvilin A and Berger A 2016 Remote Magnetomechanical Nanoactuation Small n/a - n/a

[38] Begun E, Dobrovolskiy O V, Kompaniiets M, Sachser R, Gspan C, Plank H and Huth M 2015 Post-growth purification of Co nanostructures prepared by focused electron beam induced deposition Nanotechnology 26075301

[39] Perez-Roldan M J, Tatti F, Vavassori P, Berger A and Chuvilin A 2015 Segregation of materials in double precursor electron-beam-induced-deposition: a route to functional magnetic nanostructures Nanotechnology 26375302

[40] Dobrovolskiy O V, Kompaniiets M, Sachser R, Porrati F, Gspan C, Plank H and Huth M 2015 Tunable magnetism on the lateral mesoscale by post-processing of $\mathrm{Co} / \mathrm{Pt}$ heterostructures Beilstein J. Nanotechnol. 6 1082-90

[41] Porrati F, Begun E, Sachser R and Huth M 2014 Spin-dependent transport between magnetic nanopillars through a nano-granular metal matrix J. Phys. D. Appl. Phys. 47 495001 
[42] Sangiao S, Morelln L, Ibarra M R and De Teresa J M 2011 Ferromagnet-superconductor nanocontacts grown by focused electron/ion beam techniques for current-in-plane Andreev Reflection measurements Solid State Commun. 151 37-41

[43] Nakagawa N, Hwang H Y and Muller D A 2006 Why some interfaces cannot be sharp Nat. Mater. 5 204-9

[44] Bibes M, Villegas J E and Barthélémy A 2011 Ultrathin oxide films and interfaces for electronics and spintronics Adv. Phys. 60 5-84

[45] Nogués J, Sort J, Langlais V, Skumryev V, Suriñach S, Muñoz J S and Baró M D 2005 Exchange bias in nanostructures Phys. Rep. 422 65-117

[46] Lauhon L J, Gudiksen M S, Wang D and Lieber C M 2002 Epitaxial core-shell and core-multishell nanowire heterostructures Nature 420 57-61

[47] Tang J, Huo Z, Brittman S, Gao H and Yang P 2011 Solution-processed core-shell nanowires for efficient photovoltaic cells. Nat. Nanotechnol. $6568-72$

[48] Cui L-F, Yang Y, Hsu C-M and Cui Y 2009 Carbon-Silicon Core-Shell Nanowires as High Capacity Electrode for Lithium Ion Batteries Nano Lett. $93370-4$

[49] Lu X, Yu M, Wang G, Zhai T, Xie S, Ling Y, Tong Y and Li Y 2013 H-TiO 2 @MnO 2 //H-TiO $2 @$ C Core-Shell Nanowires for High Performance and Flexible Asymmetric Supercapacitors Adv. Mater. 25 267-72

[50] Law M, Greene L E, Radenovic A, Kuykendall T, Liphardt J and Yang P 2006 ZnO-Al 2 O 3 and ZnO-TiO 2 Core-Shell Nanowire Dye-Sensitized Solar Cells J. Phys. Chem. B $11022652-63$

[51] Chong Y T, Görlitz D, Martens S, Yau M Y E, Allende S, Bachmann J and Nielsch K 2010 Multilayered core/shell nanowires displaying two distinct magnetic switching events. Adv. Mater. 22 2435-9

[52] Shi D-W, Javed K, Ali S S, Chen J-Y, Li P-S, Zhao Y-G and Han X-F 2014 Exchange- 
biased hybrid ferromagnetic-multiferroic core-shell nanostructures. Nanoscale 67215 20

[53] Wolf D, Rodriguez L A, Béché A, Javon E, Serrano L, Magen C, Gatel C, Lubk A, Lichte H, Bals S, Van Tendeloo G, Fernández-Pacheco A, De Teresa J M and Snoeck E 2015 3D Magnetic Induction Maps of Nanoscale Materials Revealed by Electron Holographic Tomography Chem. Mater. 27 6771-8

[54] Wachter S, Gavagnin M, Wanzenboeck H D, Shawrav M M, Belić D and Bertagnolli E 2014 Nitrogen as a carrier gas for regime control in focused electron beam induced deposition Nanofabrication 1

[55] Hochleitner G, Wanzenboeck H D and Bertagnolli E 2008 Electron beam induced deposition of iron nanostructures J. Vac. Sci. Technol. B Microelectron. Nanom. Struct. 26939

[56] van Dorp W F, Hansen T W, Wagner J B and De Hosson J T M 2013 The role of electron-stimulated desorption in focused electron beam induced deposition Beilstein J. Nanotechnol. 4 474-80

[57] Córdoba R, Fernández-Pacheco R, Fernández-Pacheco A, Gloter A, Magén C, Stéphan O, Ibarra M R and De Teresa J M 2011 Nanoscale chemical and structural study of Cobased FEBID structures by STEM-EELS and HRTEM. Nanoscale Res. Lett. 6592

[58] Gatel C and Snoeck E 2012 Magnetic mapping using electron holography Transmission Electron Microscopy in Micro-nanoelectronics ed A Claverie (London: ISTE-Wiley)

[59] Tang J, Wang C-Y, Jiang W, Chang L-T, Fan Y, Chan M, Wu C, Hung M-H, Liu P-H, Yang H-J, Tuan H-Y, Chen L-J and Wang K L 2012 Electrical Probing of Magnetic Phase Transition and Domain Wall Motion in Single-Crystalline Mn 5 Ge 3 Nanowire Nano Lett. 12 6372-9

[60] Liu G, Lin Y-C, Liao L, Liu L, Chen Y, Liu Y, Weiss N O, Zhou H, Huang Y and Duan 
X 2012 Domain Wall Motion in Synthetic Co 2 Si Nanowires Nano Lett. 12 1972-6

[61] Yan M, Andreas C, Kákay A, García-Sánchez F and Hertel R 2011 Fast domain wall dynamics in magnetic nanotubes: Suppression of Walker breakdown and Cherenkov-like spin wave emission Appl. Phys. Lett. 99122505 


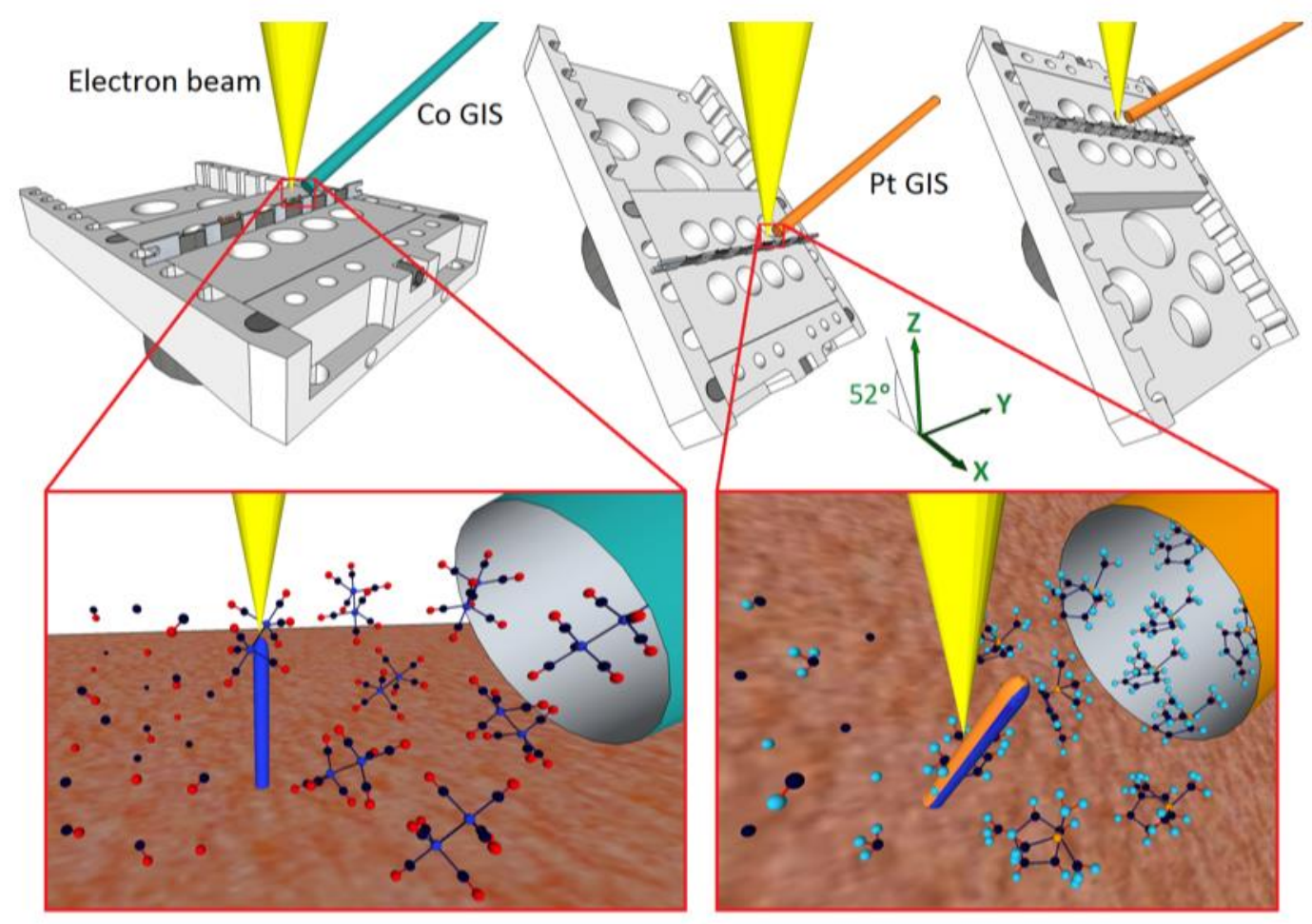

Figure 1. Diagram of the FEBID processes involved in Co core and Pt shell fabrication.
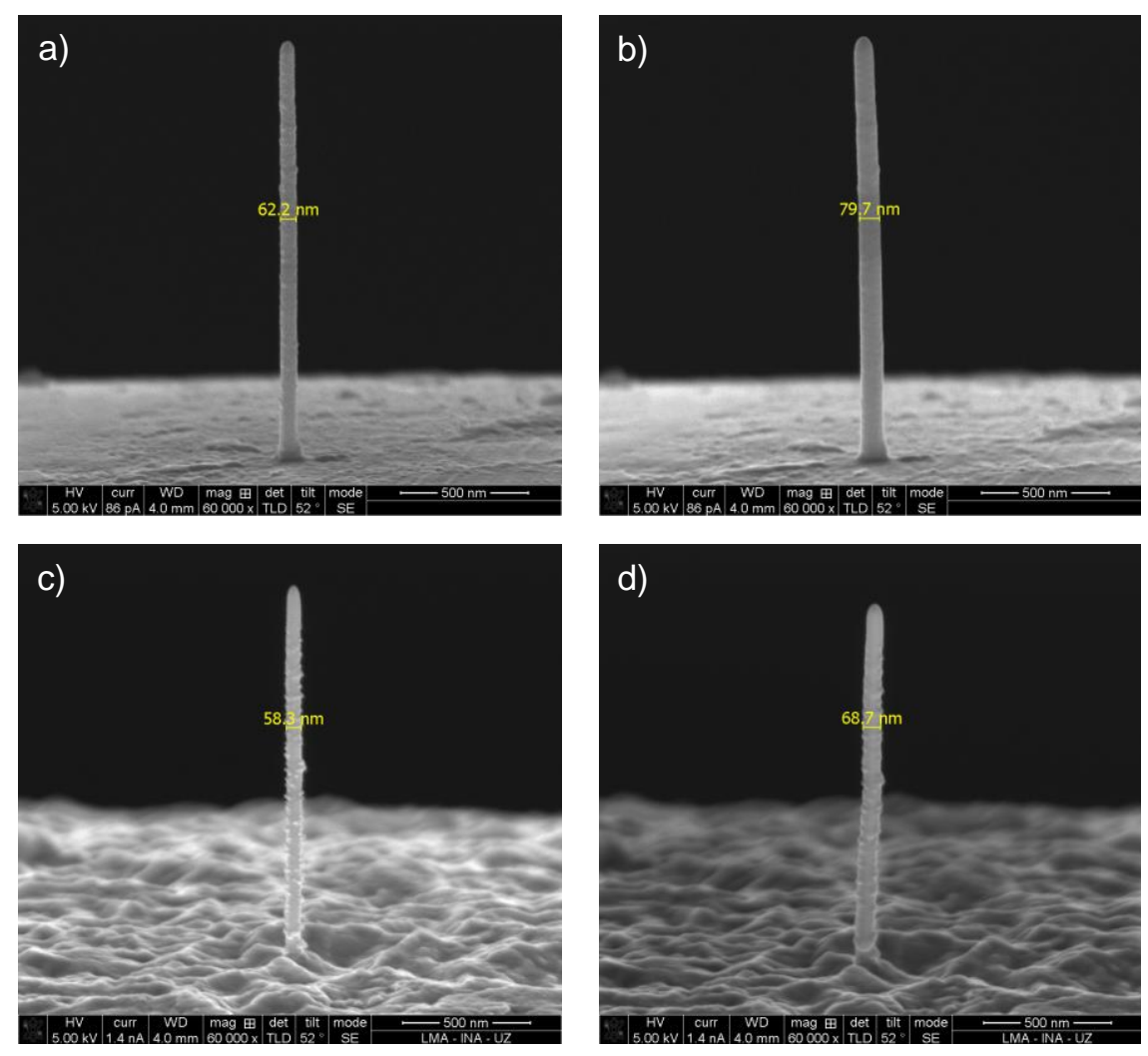

Figure 2. SEM images of (a) uncoated and (b) Pt-coated Co-FEBID nanowire, and (c) uncoated and (d) Pt-coated Fe-FEBID nanowire. 

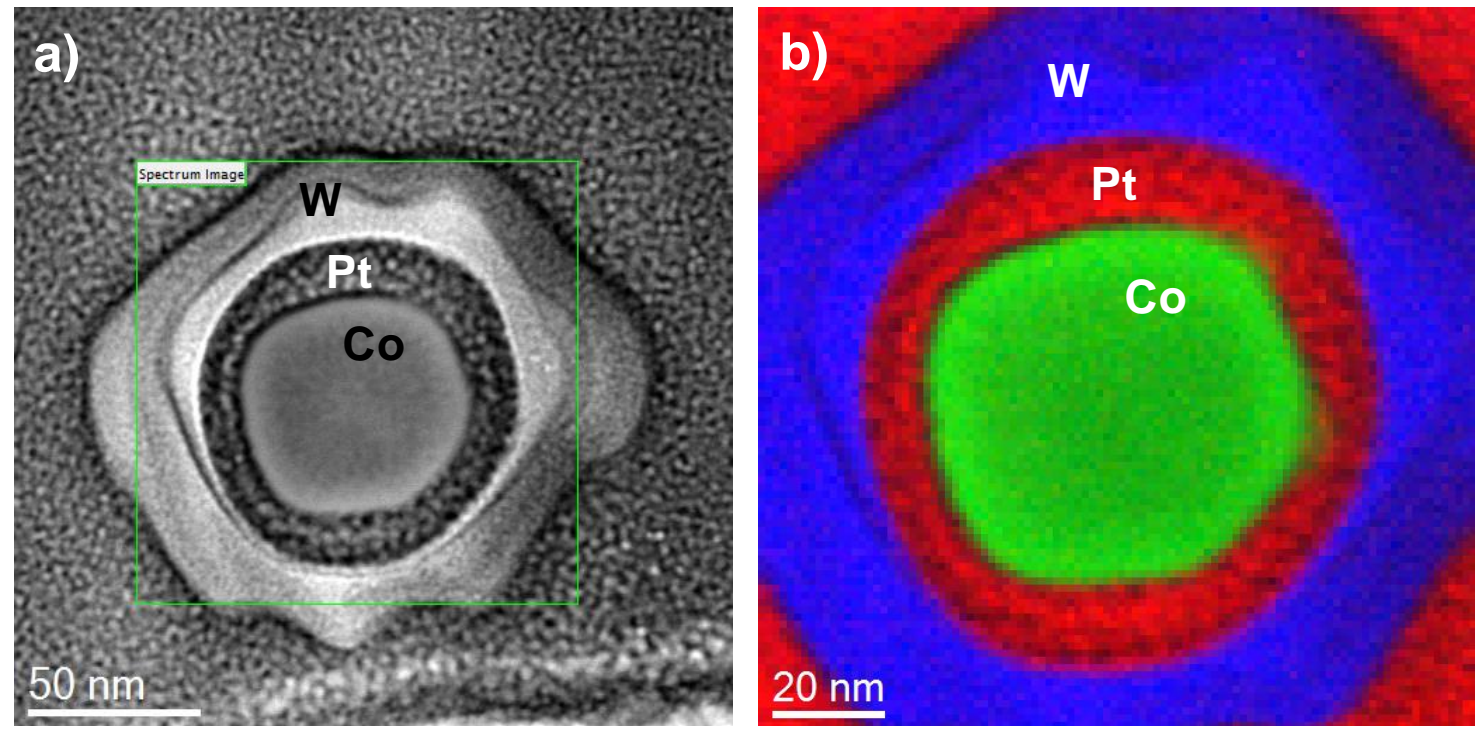

Fig. 3 Cross section of the Co-FEBID@Pt nanowire shown in Fig. 1(b). a) STEM image, b) STEM-EELS chemical color map of the region enclosed the green square in (a) where the integrated intensities of $\mathrm{Co}_{2,3}$ (in green), $\mathrm{Pt} \mathrm{M}_{4,5}$ (in red) and $\mathrm{W}_{4,5}$ (in blue) edges are depicted. 

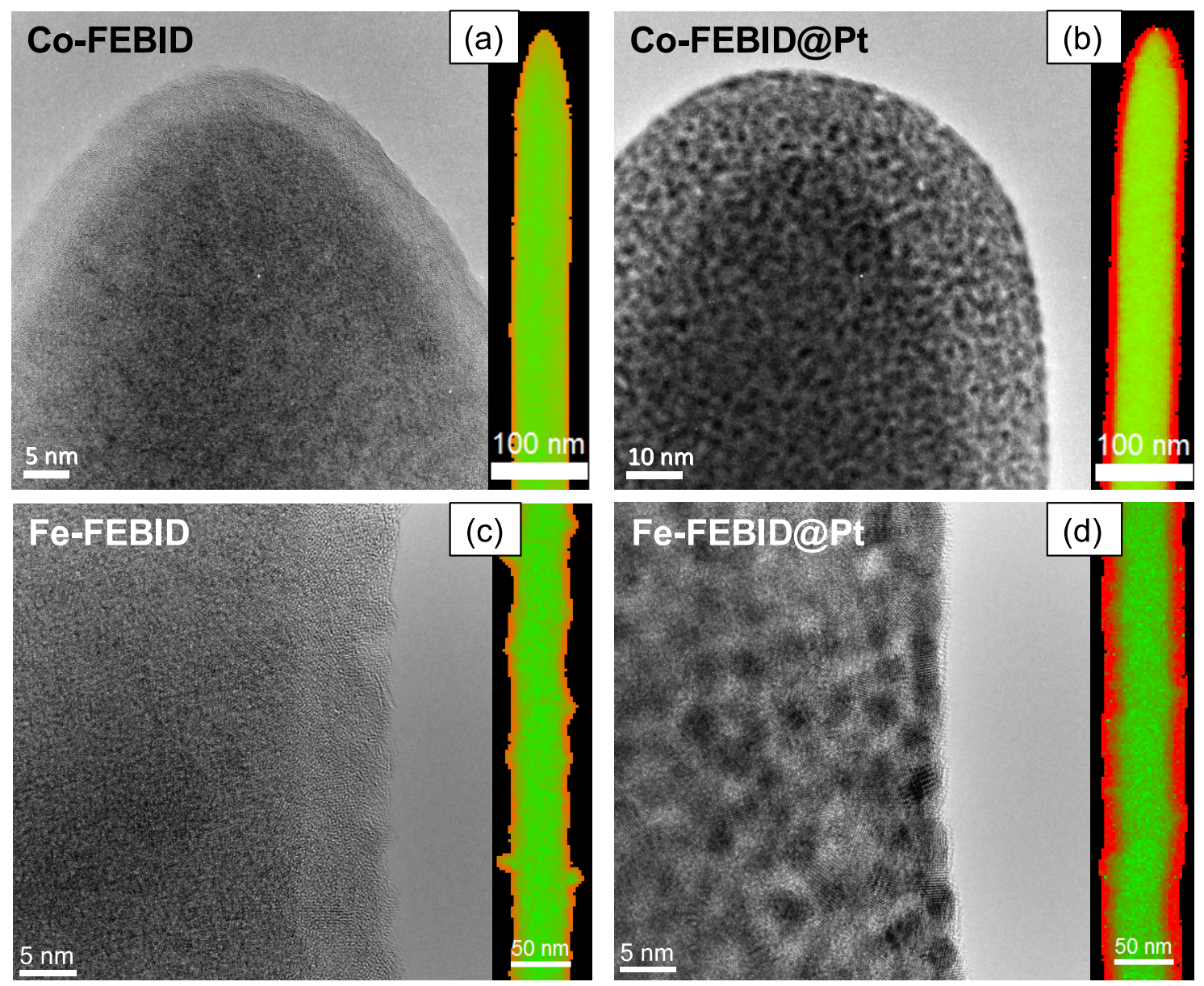

Figure 4. HRTEM images of the surface of (a) uncoated Co-FEBID, (b) core-shell CoFEBID@Pt; (c) uncoated Fe-FEBID and (d) and core-shell Fe-FEBID@Pt nanowires. Note that the core size is the same, but the image scales are changed in (b) panel to fit the Pt shell. Each image is accompanied by a color-coded STEM-EELS chemical map showing the spatial distribution of relative compositions of $\mathrm{Co}(\mathrm{Fe})$ and $\mathrm{O}$ in green and red, respectively. 

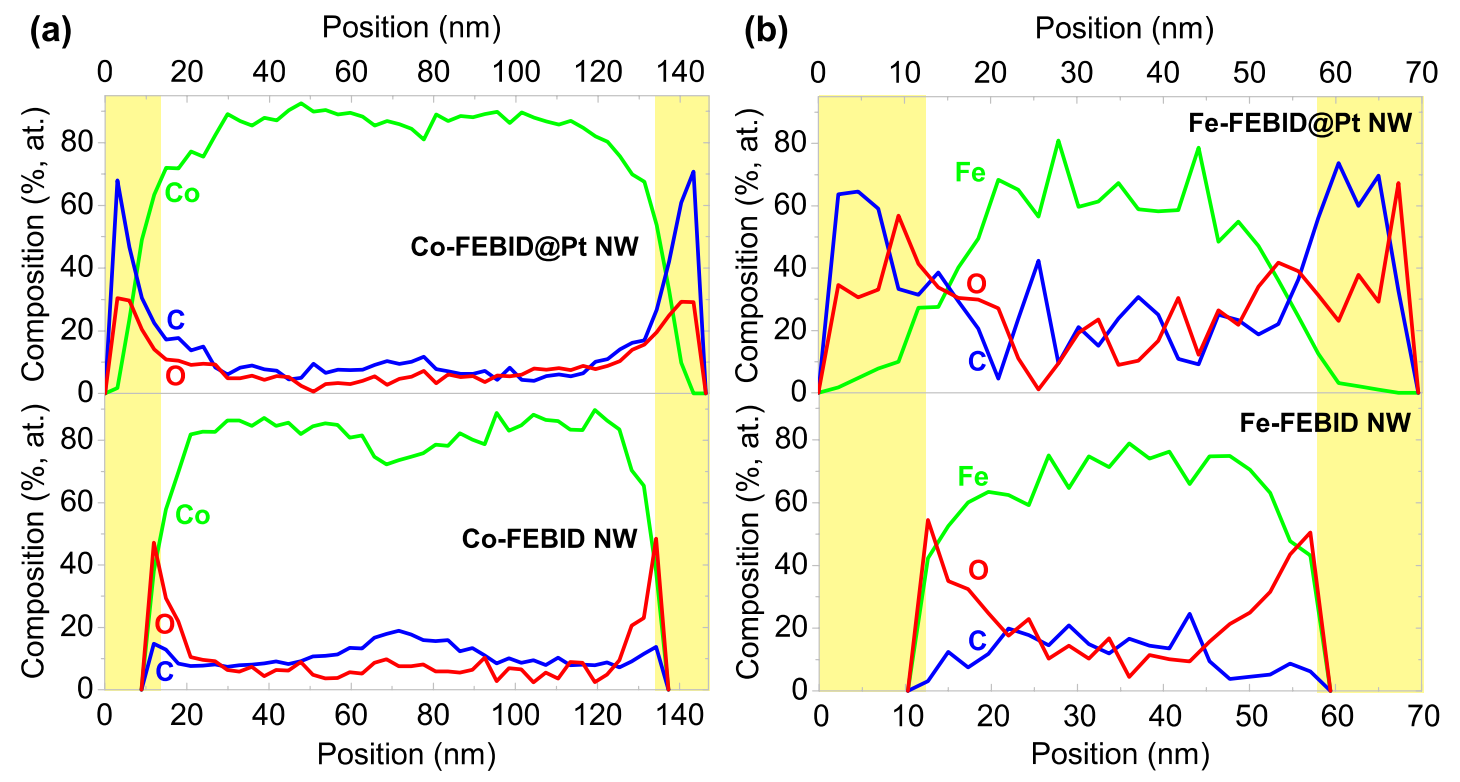

Figure 5. STEM-EELS profiles of chemical composition comparing the position of the oxide layer in Co-FEBID NW and the Co-FEBID@Pt (a); and in the Fe-FEBID NW and the FeFEBID@Pt (b). Yellow bands are guides to the eye showing the approximate position of the Pt shells. 

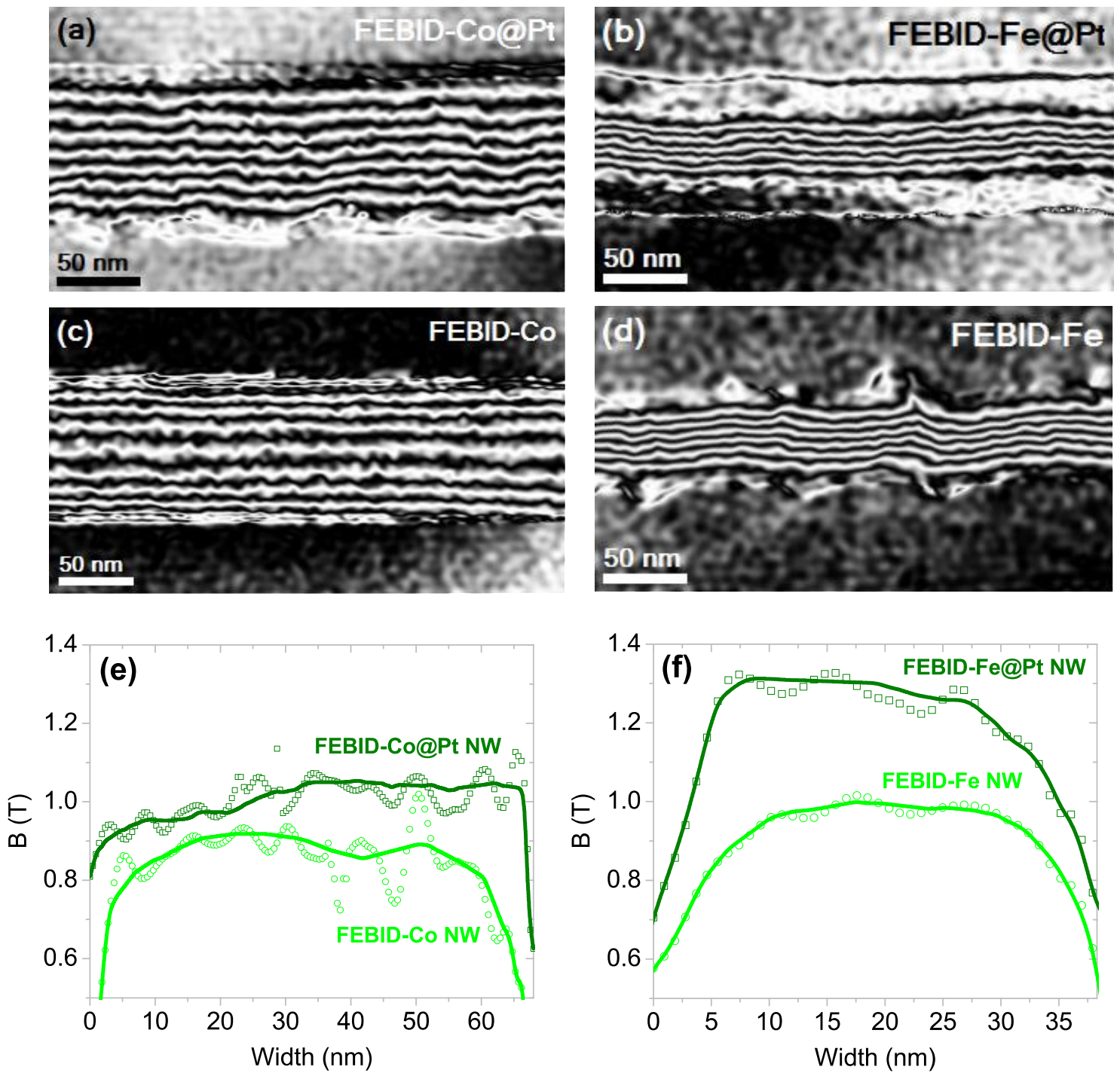

Figure 6. Magnetic flux distribution measured by off-axis electron holography of the (a) CoFEBID@Pt, (b) Fe-FEBID @Pt, (c) Co-FEBID and (d) Fe-FEBID NWs. Average magnetic induction calculated for the core-shell nanowires and their corresponding naked magnetic cores for $\mathrm{Co}(\mathrm{e})$ and $\mathrm{Fe}(\mathrm{f})$ measured across the nanowires width. 